University of Nebraska - Lincoln

DigitalCommons@University of Nebraska - Lincoln

Xia Hong Publications

Research Papers in Physics and Astronomy

2006

\title{
Effect of electric field doping on the anisotropic magnetoresistance in doped manganites
}

X. Hong

J. -B. Yau

J. D. Hoffman

C. H. Ahn

Follow this and additional works at: https://digitalcommons.unl.edu/physicshong

Part of the Atomic, Molecular and Optical Physics Commons, and the Engineering Physics Commons

This Article is brought to you for free and open access by the Research Papers in Physics and Astronomy at DigitalCommons@University of Nebraska Lincoln. It has been accepted for inclusion in Xia Hong Publications by an authorized administrator of DigitalCommons@University of Nebraska Lincoln. 


\title{
Effect of electric field doping on the anisotropic magnetoresistance in doped manganites
}

\author{
X. Hong, J.-B. Yau, J. D. Hoffman, and C. H. Ahn \\ Department of Applied Physics, Yale University, New Haven, Connecticut, USA \\ Y. Bason and L. Klein \\ Department of Physics, Bar-Ilan University, Ramat-Gan, Israel \\ (Received 22 August 2006; published 7 November 2006)
}

\begin{abstract}
We have modulated the anisotropic magnetoresistance (AMR) in 3-4 $\mathrm{nm}$ manganite films using the ferroelectric field effect-a method that electrostatically varies the carrier density without affecting the lattice distortion. While significant changes have been induced in $T_{C}$ and $\rho$, the AMR ratio remains the same when the magnetic state is not changed. This scaling behavior is in striking contrast to chemical doping results, where similar modulation of the carrier concentration $(\sim 0.1 / \mathrm{Mn})$ changes the AMR ratio by $\geqslant 30 \%$. The results reveal unambiguously the dominant role of chemical distortion in determining the AMR in manganites.
\end{abstract}

DOI: 10.1103/PhysRevB.74.174406

Anisotropic magnetoresistance (AMR) is one of the fundamental effects exhibited by magnetic metals, where the resistivity depends on the angle between the current and the magnetization. ${ }^{1}$ Arising from the mixing of spin states induced by spin-orbit coupling in rigid band exchange splitting, it contains information about the nature of impurity scattering and spin-orbit coupling in magnetic materials. ${ }^{2,3}$ This effect has proven to be a useful tool to examine ballistic transport in ferromagnetic atomic point contacts, ${ }^{4,5}$ study spin-dependent band structure in spin-valve structures, ${ }^{6}$ determine the intrinsic domain wall resistance of $180^{\circ}$ Néel walls in magnetic thin films, ${ }^{7}$ and probe the in-plane magnetocrystalline anisotropy and spin polarization in correlated electron systems. ${ }^{8-10}$

Early theoretical efforts to understand AMR focused on its manifestation in ferromagnetic $3 d$ alloys, whose Fermi surfaces contain $s$ subbands and spin-split $d$ subbands..$^{11,12}$ The framework of understanding AMR in these materials has been based on Mott's two-current model, where there are two parallel conduction channels for spin-up and spin-down electrons, respectively. ${ }^{2}$ In each channel the conduction is mainly carried by the more mobile electrons of the $s$ subbands, and the two channels are mixed via $s-d$ scattering in the presence of spin-orbit coupling. These models, which examined the normalized AMR $\left(\rho_{\|}-\rho_{\perp}\right) /\left(\rho_{\|} / 3+2 \rho_{\perp} / 3\right)$, where $\rho_{\|}$and $\rho_{\perp}$ are the resistivities with current parallel and perpendicular to the magnetization, respectively, were relatively successful in accounting for the effect of various impurities on AMR. ${ }^{13}$

The theoretical models developed for ferromagnetic $3 d$ alloys are unlikely to be applicable to strongly correlated magnetic oxides, such as the colossal magnetoresistive (CMR) materials. Their band structures are different (no $s$ subbands), and their transport properties display unusual behavior, such as high sensitivity to the presence of chemical disorder/distortion. It has been shown that strong spindependent disorder can significantly enhance the spin-mixing scattering and change the AMR effect. ${ }^{13,14}$ On the other hand, AMR in CMR materials is particularly intriguing because of the unusual interplay between the magnetic and transport properties in these compounds. Furthermore, recent observation of the giant planar Hall effect, a phenomenon closely related to the AMR effect, in manganites, and the possibility that this effect could be useful for applications
PACS number(s): 75.47.Gk

such as magnetic sensing and magnetic random access memory (MRAM) makes the elucidation of the factors determining AMR in these compounds even more relevant. ${ }^{15-18}$

The AMR in CMR materials has previously been studied in $\mathrm{La}_{1-x} \mathrm{Sr}_{x} \mathrm{MnO}_{3}$ (LSMO) and $\mathrm{La}_{1-x} \mathrm{Ca}_{x} \mathrm{MnO}_{3}$ (LCMO) thin films. The AMR ratio, which is the AMR $\left(\left|\rho_{\|}-\rho_{\perp}\right|\right)$ normalized to the zero field cooling resistivity (which we denote as $\Delta_{\mathrm{AMR}}$ ), was observed to decrease with increasing chemical doping level and magnetic Curie temperature. ${ }^{19}$ In these studies, however, the charge carriers are introduced by chemical substitution, which inherently adds chemical disorder/distortion. Here, we present an electric field-effect study of the doping dependence of AMR in CMR oxides, providing the ability to probe the independent role of charge carriers without introducing the complicating effects of substitutional disorder/distortion.

We measured LSMO films with $x=0.33,0.2$, and 0.16 , and LCMO films with $x=0.3$, and found that while the electric field doping induces significant modulation in the magnetic Curie temperature and resistivity of manganite thin films, $\Delta_{\text {AMR }}$ remains unchanged, in sharp contrast with chemical doping experiments. The striking difference between the chemical doping and electric field doping results clearly indicates that the change in carrier concentration alone cannot account for the observed changes in AMR induced by chemical doping.

The field effect modulates the carrier concentration in a clean and controlled fashion; however, for significant changes, an electric field comparable to the areal carrier density of the system is required-an extreme challenge in manganites. In the ferromagnetic metallic phase, these materials have a carrier density of $\sim 10^{21}$ carriers $/ \mathrm{cm}^{3}$ and an electronic screening length on the order of one atomic layer. ${ }^{20}$ The required polarization field to modulate the carrier density is $\sim 50 \mu \mathrm{C} / \mathrm{cm}^{2}$, which here is provided by a ferroelectric oxide, $\mathrm{Pb}\left(\mathrm{Zr}_{0.2} \mathrm{Ti}_{0.8}\right) \mathrm{O}_{3}$ (PZT). This field is an order of magnitude larger than the breakdown field of conventional dielectrics such as $\mathrm{SiO}_{2}$. The direction of the polarization field can be switched by applying a voltage pulse larger than the coercive voltage $(\sim 1 \mathrm{~V} / 1000 \AA)$ across the PZT layer with the appropriate polarity. Hence, the field effect can be modulated between a state where it increases the carrier den- 
a
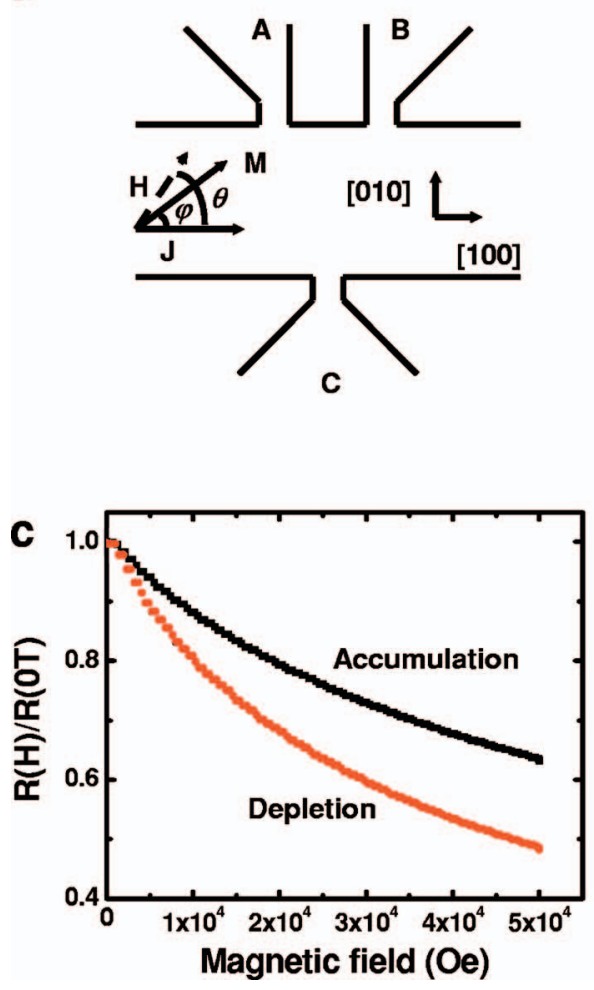
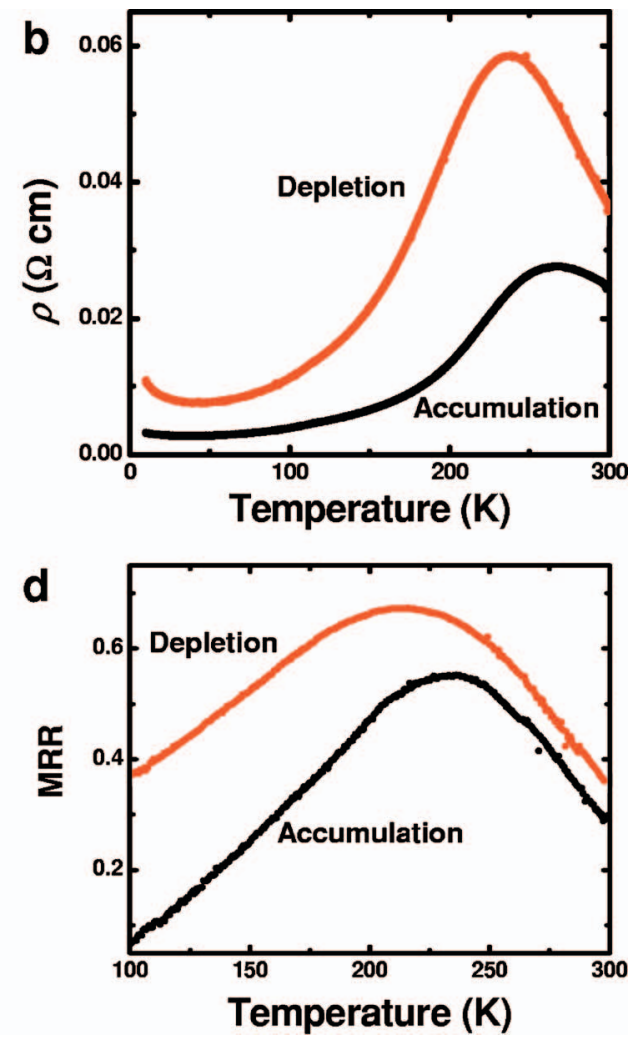

FIG. 1. (Color) (a) Hall bar configuration used for the experiment; (b) resistivity of a $3 \mathrm{~nm}$ $\mathrm{La}_{0.67} \mathrm{Sr}_{0.33} \mathrm{MnO}_{3}$ film in a PZT/ LSMO heterostructure as a function of temperature for accumulation (black) and depletion (red) states; (c) magnetoresistance as a function of magnetic field at $175 \mathrm{~K}$ in accumulation (black) and depletion (red); (d) magnetoresistance ratio at $80 \mathrm{kOe}$ as a function of temperature in accumulation (black) and depletion (red). sity in the CMR layer (accumulation state) and a state where it depletes charge carriers from the CMR layer (depletion state). Switching between accumulation and depletion states are reversible. ${ }^{20,21}$

For these experiments, we grew 3-4 nm thick LSMO and LCMO layers epitaxially on (001) $\mathrm{SrTiO}_{3}$ substrates using off-axis magnetron sputtering, then deposited 200-300 nm thick PZT layers in situ on top of the CMR layers. We have obtained films with a high degree of crystallinity and atomically smooth surfaces, which are important parameters since the field effect occurs exclusively at the atomic-scale interface between the two materials. The details of growth and structural and surface characterization are discussed elsewhere. ${ }^{20,21}$ X-ray diffraction measurements show that CMR films of these thicknesses are strained, with an in-plane lattice constant of $3.90 \AA$ and a $c$-axis lattice constant of $3.84 \AA$ A. Transmission electron microscopy measurements reveal high quality samples and no signs of a prevalence of grain boundaries or other defects.

The samples were patterned into Hall bars, with dimensions ranging from $10 \mu \mathrm{m}$ to $100 \mu \mathrm{m}$, and gold electrodes were deposited for transport measurements. The current was applied along one of the in-plane crystal axes, [100] or [010], while a constant magnetic field was applied in the plane of the film, making an angle $\theta$ to the current direction [Fig. $1(\mathrm{a})]$.

In the chemically doped LSMO films, $\Delta_{\mathrm{AMR}}$ decreases with increasing doping level. For example, the maximum $\Delta_{\mathrm{AMR}}$ at $7 \mathrm{kOe}$ in single layer $\mathrm{La}_{0.67} \mathrm{Sr}_{0.33} \mathrm{MnO}_{3}$ films is $\sim 30 \%$ smaller than in $\mathrm{La}_{0.8} \mathrm{Sr}_{0.2} \mathrm{MnO}_{3}$ films, ${ }^{19}$ and larger changes have been observed at lower magnetic fields. To compare this result with electric field-effect doping, we have used $\mathrm{PZT} / \mathrm{La}_{0.67} \mathrm{Sr}_{0.33} \mathrm{MnO}_{3}$ heterostructures where the LSMO layer is $3 \mathrm{~nm}$ thick. For this composition, films as thin as $3 \mathrm{~nm}$ maintain low-temperature metallic behavior, while thinner films $(2-2.5 \mathrm{~nm})$ are insulating over the entire temperature range. ${ }^{20}$ Sawyer-Tower measurements of these heterostructures show a polarization of $\sim 50 \mu \mathrm{C} / \mathrm{cm}^{2}$, and Hall effect measurements yield estimated carrier densities of $\sim 0.3$ carriers/unit cell (u.c.) in the accumulation state and $\sim 0.2$ carriers/unit cell (u.c.) in the depletion state. ${ }^{23}$ Figure 1(b) shows the temperature dependence of the resistivity of one of these films. The lower curve, with a peak in the resistivity at $\sim 265 \mathrm{~K}$, corresponds to the accumulation state, while the upper curve, with a peak temperature of $\sim 240 \mathrm{~K}$, corresponds to the depletion state. Concomitantly, the mag-

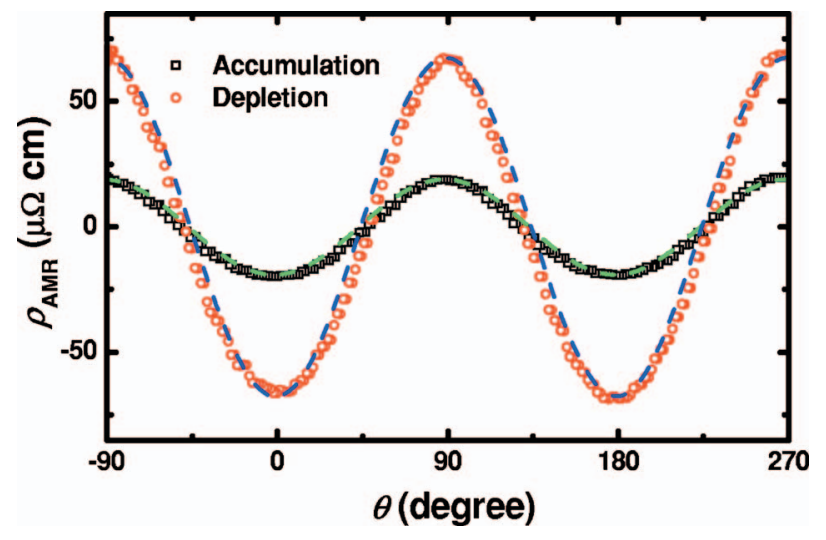

FIG. 2. (Color) The angular dependence of $\rho_{\mathrm{AMR}}$ at $150 \mathrm{~K}$ and $20 \mathrm{kOe}$ (symbols) for accumulation (black) and depletion (red) states, along with fits to $\cos 2 \theta$ (dashed lines). 


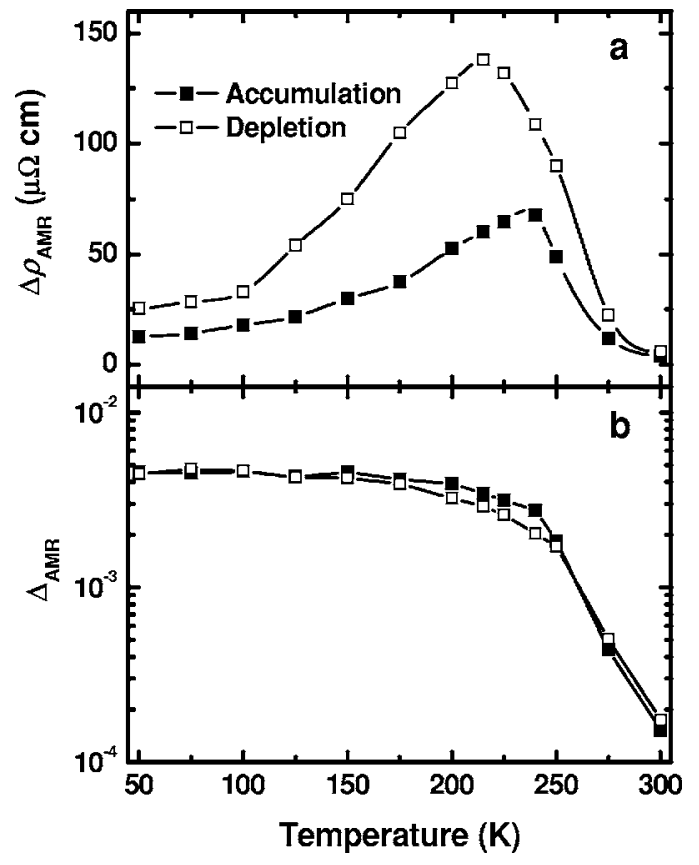

FIG. 3. (a) $\Delta \rho_{\text {AMR }}$ as a function of temperature for accumulation (solid) and depletion (open) states; (b) $\Delta_{\mathrm{AMR}}$ at $20 \mathrm{kOe}$ as a function of temperature for accumulation (solid) and depletion (open) states.

netoresistance is enhanced in the low-carrier density state, with the magnetoresistance ratio $\{\mathrm{MRR}=[R(0)$ $-R(80 \mathrm{kOe})] / R(0\}$ increasing from $55 \%$ at $235 \mathrm{~K}$ in accumulation to $67 \%$ at $210 \mathrm{~K}$ in depletion [Figs. 1(c) and 1(d)]. These changes are consistent with previous results observed in chemical doping and field-effect experiments. ${ }^{21,22}$

We now turn to investigate the effect of field-effect induced changes in carrier density on the AMR. When the magnetization makes an angle $\varphi$ with the current direction, the resistivity $\rho$ has the angular dependence: ${ }^{1}$

$$
\rho=\frac{1}{2}\left(\rho_{\|}+\rho_{\perp}\right)+\frac{1}{2}\left(\rho_{\|}-\rho_{\perp}\right) \cos 2 \varphi=\frac{1}{2}\left(\rho_{\|}+\rho_{\perp}\right)+\rho_{A M R} .
$$

Figure 2 shows the angular dependence of $\rho_{\mathrm{AMR}}$ at $150 \mathrm{~K}$ (below $\left.T_{\mathrm{C}}\right)$. At high-magnetic fields $(20 \mathrm{kOe})$, the magnetization follows the field direction $(\varphi=\theta)$, and $\rho_{\text {AMR }}$ shows the expected $\cos 2 \theta$ angular dependence. Figure 3(a) shows the peak-to-peak amplitude, $\Delta \rho_{\mathrm{AMR}}=\left|\rho_{\|}-\rho_{\perp}\right|$, at $20 \mathrm{kOe}$ as a function of temperature. $\Delta \rho_{\mathrm{AMR}}$ is enhanced in the depletion state, and in both accumulation and depletion states, $\Delta \rho_{\text {AMR }}$ peaks at a temperature about $30 \mathrm{~K}$ below its corresponding $T_{C}$, coinciding with the MRR peak temperatures.

Figure 3(b) shows the temperature dependence of $\Delta_{\mathrm{AMR}}$. Strikingly, we find that $\Delta_{\mathrm{AMR}}$ for the accumulation and depletion states collapse onto each other. This scaling behavior with electric field doping suggests that $\Delta_{\mathrm{AMR}}$ is independent of carrier concentration, in sharp contrast to the chemical doping results, even though the changes of the doping level are similar in both experiments.

To understand how this scaling with electric field doping

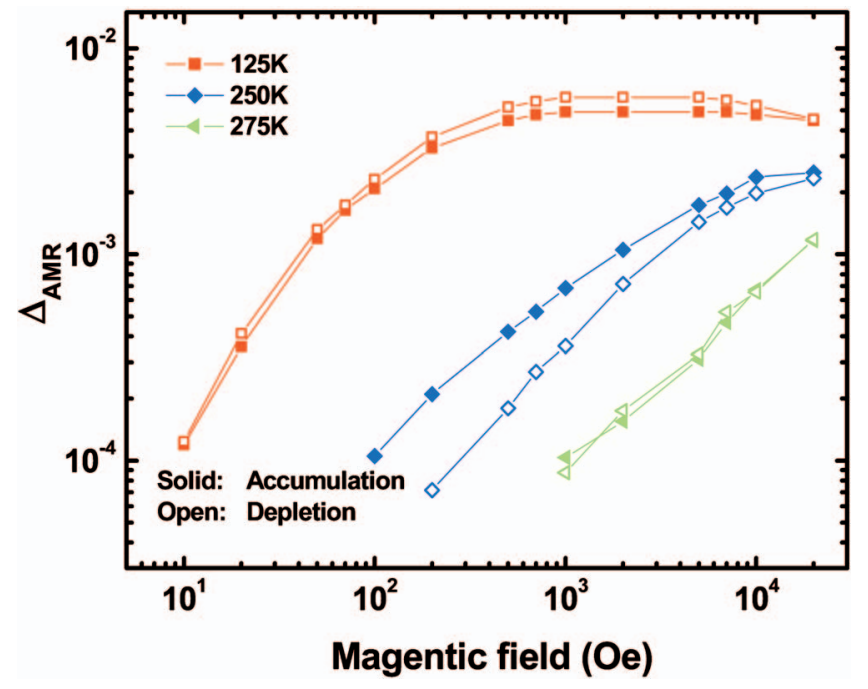

FIG. 4. (Color) Field dependence of $\Delta_{\mathrm{AMR}}$ for accumulation (solid) and depletion (open) states at $125 \mathrm{~K}$ (red squares), $250 \mathrm{~K}$ (blue diamonds), and $275 \mathrm{~K}$ (green triangles).

is related to magnetization, we measured $\Delta_{\mathrm{AMR}}$ in different magnetic states of the sample. Figure 4 shows the magnetic field dependence of $\Delta_{\mathrm{AMR}}$ at various temperatures. At $125 \mathrm{~K}$, well below $T_{\mathrm{C}}, \Delta_{\mathrm{AMR}}$ scales with the carrier densities in magnetic fields from 10 Oe to $20 \mathrm{kOe}$. This behavior is observed up to $240 \mathrm{~K}$, where the sample is ferromagnetic in both states. At $275 \mathrm{~K}$, where the sample is paramagnetic for both polarization directions of the PZT layer, the scaling of $\Delta_{\mathrm{AMR}}$ is also maintained. However, at $250 \mathrm{~K}$, where the sample is ferromagnetic in accumulation but paramagnetic in depletion, $\Delta_{\mathrm{AMR}}$ shows different magnetic field dependences.

Given that the saturation magnetization of $\mathrm{La}_{1-x} \mathrm{Sr}_{x} \mathrm{MnO}_{3}$ is $4-x \mu_{\mathrm{B}}$ /u.c., and the electric field changes the carrier density by 0.1 carriers/u.c., the field effect only induces a $3 \%$ change in the saturation magnetization. The scaling of $\Delta_{\mathrm{AMR}}$ at high and low temperatures shows that $\Delta_{\mathrm{AMR}}$ is indepen-

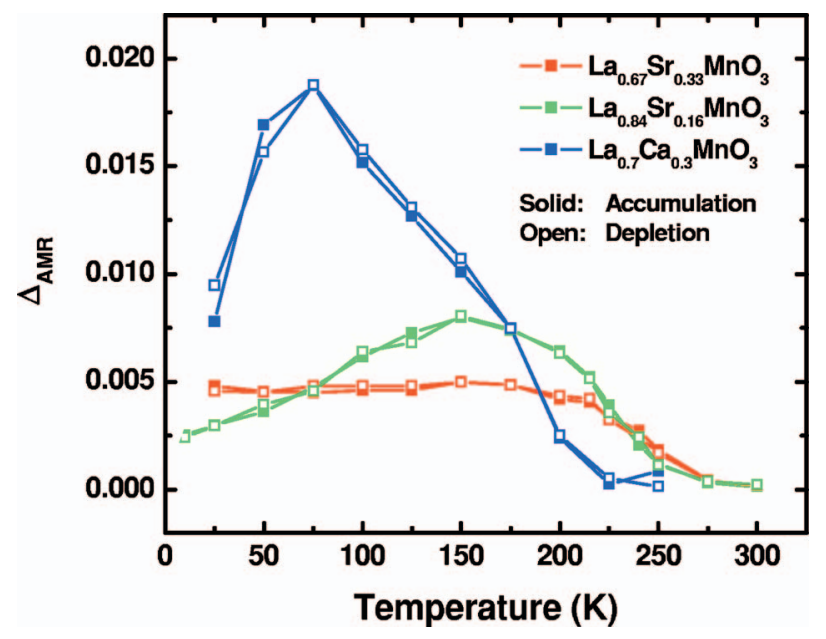

FIG. 5. (Color) Temperature dependence of $\Delta_{\mathrm{AMR}}$ at $7 \mathrm{kOe}$ for accumulation (solid) and depletion (open) states taken on LSMO heterostructures with $x=0.33$ (red) and 0.16 (green), and a $\mathrm{La}_{0.7} \mathrm{Ca}_{0.3} \mathrm{MnO}_{3}$ heterostructure (blue); 


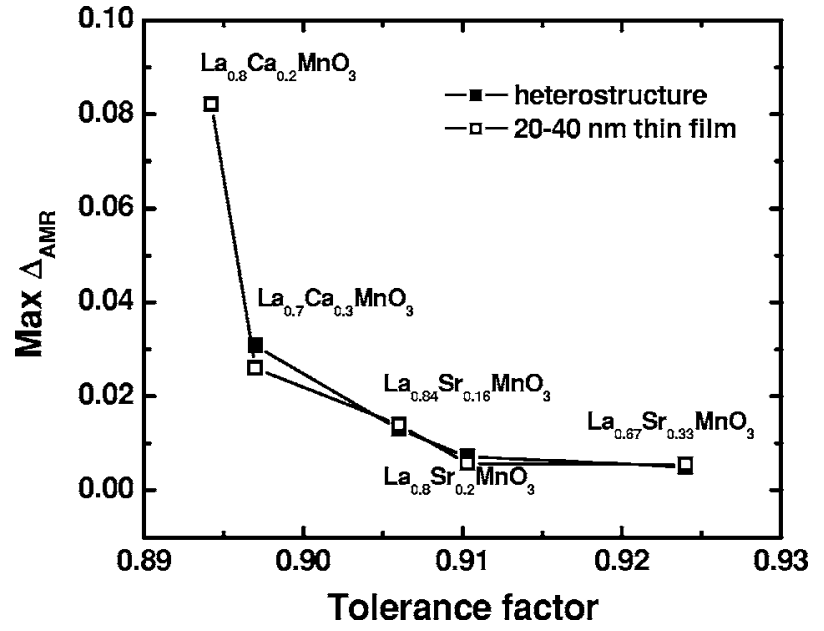

FIG. 6. Maximum $\Delta_{\mathrm{AMR}}$ measured as a function of the tolerance factor for LSMO and LCMO heterostructures (solid) and single layer films (open).

dent of doping level when the change of magnetization is small. Significant changes are only observed at temperatures between $240 \mathrm{~K}$ and $265 \mathrm{~K}$, where the field effect modulates the sample between paramagnetic and ferromagnetic states, causing large changes in magnetization.

To investigate whether the scaling behavior is generic to the CMR system, we studied the electric field effect in manganite films with different chemical doping levels and different dopants. Figure 5 shows $\Delta_{\mathrm{AMR}}$ at $7 \mathrm{kOe}$ as a function of temperature for both polarization states in three types of heterostructures: LSMO with $x=0.33$ and 0.16 , and LCMO with $x=0.3$. All three heterostructures show scaling of $\Delta_{\mathrm{AMR}}$ in magnetic fields up to $50 \mathrm{kOe}$. Compared with the LSMO $x$ $=0.33$ sample, $\Delta_{\mathrm{AMR}}$ is greatly enhanced in both the LSMO $x=0.16$ sample (with the same dopant but lower doping level) and the LCMO $x=0.3$ sample (with similar doping level but the dopant changed to $\mathrm{Ca}$ ). It is important to note that the scaling holds for a variety of manganites that differ significantly also in their degrees of phase separation, thus strengthening the evidence for the intrinsic nature of the observed scaling behavior of AMR. ${ }^{24}$

Another way in which field-effect doping differs from chemical doping is in its effect on magnetic anisotropy. The LSMO thin films show an in-plane biaxial anisotropy with a superimposed uniaxial anisotropy. ${ }^{25}$ Above the anisotropy fields, one observes the $\cos 2 \theta$ angular dependence, showing that on a macroscopic scale the magnetization is aligned with the applied magnetic field. At low magnetic fields, where the anisotropy energy dominates, the magnetization does not fully follow the field direction, and the angular dependence deviates from the sinusoidal angular dependence. We find that the minimum fields required to obtain the $\cos 2 \theta$ angular dependence at $125 \mathrm{~K}$ are $\sim 2 \mathrm{kOe}, 7 \mathrm{kOe}$, and $10 \mathrm{kOe}$, for the LSMO $x=0.33,0.16$, and LCMO $x=0.3$ samples, respectively, showing that the magnetic anisotropy changes significantly with chemical doping. ${ }^{26}$ In sharp contrast, there are no appreciable differences in these field values between the two polarization states in the electric field-effect experiments.

A plausible reason for these differences is that with the electric field effect, one only changes the fraction of $\mathrm{Mn}^{3+} / \mathrm{Mn}^{4+}$ ions without introducing lattice distortion, while in the chemical doping approach, one changes the doping level by replacing trivalent $\mathrm{La}$ by divalent $\mathrm{Sr}$ or $\mathrm{Ca}$. Adding the divalent element relaxes the Jahn-Teller distortion present in the $\mathrm{Mn}^{3+}$ ions, as well as the crystal lattice distortion due to the size difference between $\mathrm{Sr}^{2+}, \mathrm{Ca}^{2+}$, and $\mathrm{La}^{3+} \cdot{ }^{27}$ This distortion changes the $\mathrm{O}-\mathrm{Mn}-\mathrm{O}$ bonding angle, modifying the conduction bandwidth and the spin orbit coupling.

To quantify these results in more detail, we consider the distortion level of the perovskites, which can be characterized by the tolerance factor, $t=\left(r_{A}+r_{\mathrm{O}}\right) / \sqrt{ } 2\left(r_{\mathrm{Mn}}+r_{\mathrm{O}}\right)$. Using a tolerance factor that is the weighted average of the two $A$-site ions, we calculated the tolerance factors of LSMO and LCMO with different doping levels. ${ }^{27}$ We also carried out a comprehensive AMR study in LSMO and LCMO films and heterostructures, in magnetic fields ranging from $10 \mathrm{Oe}$ to $50 \mathrm{kOe}$, and in temperatures from $5 \mathrm{~K}$ to $300 \mathrm{~K}$, to find the maximum AMR ratio. Figure 6 shows the maximum $\Delta_{\text {AMR }}$ of $\mathrm{La}_{1-x} \mathrm{Sr}_{x} \mathrm{MnO}_{3}$ heterostructures with $x=0.33$ $(3 \mathrm{~nm}), 0.2(4 \mathrm{~nm})$, and $0.16(4 \mathrm{~nm})$, and $\mathrm{La}_{1-x} \mathrm{Ca}_{x} \mathrm{MnO}_{3}$ heterostructures with $x=0.3(4.5 \mathrm{~nm})$ as a function of the tolerance factor. Both the temperature dependence and the magnitude of the AMR obtained on relaxed single-layer manganite films $(20-40 \mathrm{~nm})$ are comparable to those observed in the 3-4 $\mathrm{nm}$ heterostructures. We also observe that the CMR heterostructures display similar AMR and resistivity values as single-layer films with the same thickness. The largest $\Delta_{\mathrm{AMR}}$ is observed in the most distorted LCMO samples. Combined with the field-effect scaling behavior observed in the heterostructures, these results demonstrate how $\Delta_{\mathrm{AMR}}$ decreases with smaller distortion, revealing the dominant effect of lattice distortion.

In conclusion, these electric field-effect experiments provide a unique way of deconvolving the contributions of carrier density and chemical disorder/distortion to the AMR effect, thereby allowing one to identify the dominant role of chemical distortion in determining the AMR ratio in doped manganites. It also allows one to induce controlled increases of $T_{\mathrm{C}}$ in manganite films without compromising the AMR, an effect that can be used to optimize properties for magnetic applications, such as MRAM devices based on the planar Hall effect. ${ }^{18}$

C.A. acknowledges primary support from the National Science Foundation under Contract Nos. MRSEC DMR 0520495 and DMR 0134721 and ONR, along with support from the Packard and Sloan Foundations. L.K. acknowledges support by the Israel Science Foundation founded by the Israel Academy of Sciences and Humanities. C.A. and L.K. acknowledge support from Grant No. 2002384 from the United States-Israel Binational Science Foundation, Jerusalem, Israel. 
${ }^{1}$ T. R. McGuire and R. I. Potter, IEEE Trans. Magn. 11, 1018 (1975).

${ }^{2}$ J. Smit, Physica (Utrecht) 16, 612 (1951).

${ }^{3}$ E. Dan Dahlberg, K. Riggs, and G. A. Prinz, J. Appl. Phys. 63, 4270 (1988).

${ }^{4}$ J. Velev, R. F. Sabirianov, S. S. Jaswal, and E. Y. Tsymbal, Phys. Rev. Lett. 94, 127203 (2005).

${ }^{5}$ M. Viret, S. Berger, M. Gabureac, F. Ott, D. Olligs, I. Petej, J. F. Gregg, C. Fermon, G. Francinet, and G. LeGoff, Phys. Rev. B 66, 220401(R) (2002).

${ }^{6}$ I. Zutic, J. Fabian, and S. Das Sarma, Rev. Mod. Phys. 76, 323 (2004).

${ }^{7}$ D. Buntinx, S. Brems, A. Volodin, K. Temst, and C. Van Haesendonck, Phys. Rev. Lett. 94, 017204 (2005).

${ }^{8}$ I. Genish, Y. Kats, L. Klein, J. W. Reiner, and M. R. Beasley, J. Appl. Phys. 95, 6681 (2004).

${ }^{9}$ J. N. Eckstein, I. Bozovic, J. O'Donnell, M. Onellion, and M. S. Rzchowski, Appl. Phys. Lett. 69, 1312 (1996).

${ }^{10}$ M. Ziese, Phys. Rev. B 62, 1044 (2000).

${ }^{11}$ I. A. Campbell, A. Fert, and O. Jaoul, J. Phys. C 3, S95 (1970).

${ }^{12}$ I. A. Campbell, J. Phys. F: Met. Phys. 4, L181 (1974).

${ }^{13}$ A. P. Malozemoff, Phys. Rev. B 32, 6080 (1985).

${ }^{14}$ J. Banhart, H. Ebert, and A. Vernes, Phys. Rev. B 56, 10165 (1997).
${ }^{15}$ H. X. Tang, R. K. Kawakami, D. D. Awschalom, and M. L. Roukes, Phys. Rev. Lett. 90, 107201 (2003).

${ }^{16}$ H. X. Tang, S. Masmanidis, R. K. Kawakami, D. D. Awschalom, and M. L. Roukes, Nature 431, 52 (2004).

${ }^{17}$ Y. Bason, L. Klein, J.-B Yau, X. Hong, and C. H. Ahn, Appl. Phys. Lett. 84, 2593 (2004)

${ }^{18}$ Y. Bason, L. Klein, J.-B Yau, X. Hong, J. D. Hoffman, and C. H. Ahn, J. Appl. Phys. 99, 08R701 (2006).

${ }^{19}$ J.-B Yau, X. Hong, C. H. Ahn, Y. Bason, and L. Klein (unpublished).

${ }^{20}$ X. Hong, A. Posadas, and C. H. Ahn, Appl. Phys. Lett. 86, 142501 (2005).

${ }^{21}$ X. Hong, A. Posadas, A. Lin, and C. H. Ahn, Phys. Rev. B 68, 134415 (2003).

${ }^{22}$ A. Urushibara, Y. Moritomo, T. Arima, A. Asamitsu, G. Kido, and Y. Tokura, Phys. Rev. B 51, 14103 (1995).

${ }^{23}$ X. Hong, Ph.D. dissertation, Yale University, 2006.

${ }^{24}$ M. Bibes, O. Gorbenko, B. Martínez, A. Kaul, and J. Fontcuberta, J. Magn. Magn. Mater. 211, 47 (2000).

${ }^{25}$ Y. Bason, L. Klein, J.-B. Yau, X. Hong, and C. H. Ahn, Phys. Status Solidi C 1, 3336 (2004).

${ }^{26}$ J.-P. Renard and M. Velázquez, Eur. Phys. J. B 34, 41 (2003).

${ }^{27}$ E. Dagotto, Nanoscale Phase Separation and Colossal Magnetoresistance (Springer-Verlag, New York, 2003). 